\title{
Perfil epidemiológico de recém-nascidos atendidos em uma maternidade de alto risco no Sul do Brasil
}

\author{
Aldo Guilherme Pretti Gesser ${ }^{a^{*}}$, Deisi Vargas ${ }^{a, b}$, Tarcisio Lins Arcoverde ${ }^{a, b}$, \\ Andressa Saorim $^{\mathrm{b}}$, Fernanda Amorim Steingräber ${ }^{\mathrm{b}}$, Gabriela Yuri Stinghen ${ }^{\mathrm{b}}$, \\ Giulia Giacomini ${ }^{\mathrm{b}}$, Alexandre Ademar Hoeller ${ }^{\mathrm{c}, \mathrm{d}}$
}

${ }^{a}$ Programa de Residência Médica em Pediatria, Fundação Hospitalar de Blumenau, Hospital Santo Antônio, Blumenau, SC, Brasil.

${ }^{\mathrm{b}}$ Universidade Regional de Blumenau (FURB), Blumenau, SC, Brasil.

${ }^{\mathrm{c}}$ Universidade do Sul de Santa Catarina (UNISUL), Palhoça, SC, Brasil.

${ }^{\mathrm{d}}$ Laboratório de Ciências Médicas, Universidade Federal de Santa Catarina (UFSC), Florianópolis, SC, Brasil.

Histórico do Artigo

Recebido em:

10/04/2019

Aceito em:

$19 / 08 / 2019$

Palavras-chave: maternidade; gravidez na adolescência; prematuridade; gestação de alto risco

\section{RESUMO}

$\mathrm{O}$ cuidado adequado ao recém-nascido representa um grande desafio para a redução dos índices de mortalidade infantil no Brasil. O presente trabalho investigou o perfil epidemiológico dos recém-nascidos em uma maternidade referência de alto risco no Sul do Brasil. Trata-se de um estudo transversal, retrospectivo, com 3.318 recém-nascidos entre julho de 2017 e junho de 2018, atendidos no centro obstétrico de um serviço referência do SUS em alta complexidade de saúde Materno-Infantil. A maioria dos recém-nascidos era do sexo masculino, com atendimento pelo SUS em $89,33 \%$ dos casos, nasceu de parto vaginal $(61,54 \%)$, a termo (idade gestacional média de 269,97 dias), com peso variando entre 485 e $5320 \mathrm{~g}$, e foi encaminhada ao alojamento conjunto $(89,72 \%)$. Em relação às gestantes, $10,7 \%$ eram adolescentes e a maioria era primigesta $(41,17 \%)$. A taxa de prematuridade foi de $17,02 \%$. O conhecimento destes dados expressa as condições de nascimento neonatais e mostram como a prematuridade é incidente em nosso meio, fazendo-se necessária elaboração de estratégias de melhoria na assistência ao pré e pós-natal de recémnascidos de alto risco.

$$
\begin{aligned}
& \text { Epidemiological profile of newborns attended at a high-risk maternity } \\
& \text { hospital in the South of Brazil }
\end{aligned}
$$

Keywords: maternity; teenage pregnancy; prematurity; high-risk gestation

\section{ABSTRACT}

The adequate care for the newborn has been one of the challenges in reducing infant mortality rates in Brazil. We aimed to describe the epidemiological profile of newborns in a high-risk reference maternity hospital in the South of Brazil. A cross-sectional retrospective study with 3.318 newborns evaluated between July 2017 and June 2018, attended at the obstetric center of a SUS referral service with a high complexity of maternal and child health care. Most of the newborns were male, attended by SUS in $89.3 \%$ of the cases, conceived by vaginal delivery (61.5\%), term (mean gestational age of 269,97 days), with weight ranging between 485 and 5320 $\mathrm{g}$, and referred to the joint accommodation $(89.7 \%)$. In relation to pregnant women, $10.7 \%$ were adolescents and the majority were primigestant $(41.2 \%)$. The prematurity rate was of $17 \%$. The present data expresses the neonatal birth conditions of a representative population and show how prematurity is incident in our environment, making it necessary to elaborate strategies to improve pre- and post-natal care of high-risk newborns.

\section{Introdução}

As taxas de morbimortalidade neonatais no Brasil são consideradas excessivas pela Organização Mundial da Saúde (OMS) (1). A mortalidade neonatal é responsável por $70 \%$ das mortes no primeiro ano de vida, e o cuidado adequado ao recém-nascido tem sido um dos desafios para a redução dos índices de mortalidade infantil no Brasil (1). Segundo Lansky e colegas, 41\% dos óbitos neonatais em 2009 (com relação a todos os óbitos de crianças com menos de cinco anos), correspondem a 3,3 milhões de óbitos em

\footnotetext{
*Autor correspondente: aldogesserped@gmail.com (Gesser A.G.P)
} 
crianças com menos de 28 dias. Entre 1990 e 2009, as taxas globais de mortalidade neonatal caíram 28\%, passando de 33,2/1.000 para 23,9/1.000 durante o período. No Brasil, a taxa de mortalidade neonatal foi de 11,1/1.000 em 2011-2012 (2).

Apesar dos avanços tecnológicos nas últimas décadas a prematuridade segue como a maior causa de morte neonatal e a segunda causa de mortalidade em crianças menores de 5 anos de vida (3). Bebês prematuros, devido ao seu desenvolvimento fetal incompleto, estão mais sujeitos a infecções, e devido à imaturidade do seu sistema imunológico, estão mais susceptíveis a distúrbios metabólicos, respiratórios, no controle da regulação da temperatura corporal, bem como dificuldade de se alimentar e necessidade de nutrição parenteral. Além disso, os prematuros podem ter maior necessidade de reanimação neonatal em sala de parto (4). É considerada prematura a criança que nasce com menos de 37 semanas de idade gestacional (3). Os bebês prematuros têm maior risco de adoecer e morrer devido ao seu desenvolvimento fetal incompleto e sua suscetibilidade a infecções (4).

Um estudo realizado em 184 países no ano de 2010 estimou o nascimento de cerca de 14,9 milhões de prematuros, o que representa $11,1 \%$ dos nascidos vivos no mundo, variando entre $5 \%$ em países europeus a $18 \%$ em países africanos (5).

Conforme estudo de Lansky e colaboradores (2014) existe uma prevalência de 11,3\% de prematuros no país, tendo este agravo contribuído para $30 \%$ das mortes neonatais. No ano de 2010, em Santa Catarina, 12\% dos recém-nascidos eram prematuros (6). Nas últimas décadas, diversas estratégias foram desenvolvidas no Brasil em busca de uma melhor atenção à saúde materno-infantil. A assistência ao pré-natal tem hoje metas extremamente amplas, especialmente no reconhecimento da gestação de alto risco, além de sua finalidade psicológica, educacional e social. A determinação dos fatores de risco perinatais exige o conhecimento da história natural das complicações da gravidez e do parto.

A manutenção e a melhoria da saúde materno-infantil são alguns dos objetivos definidos pelo Ministério da Saúde e, para isto, é essencial a atenção pré-natal e puerperal (7). O conhecimento do perfil epidemiológico perinatal proporciona subsídio para o desenvolvimento de propostas com vistas a melhorar a assistência à saúde e a organização do cuidado materno-infantil. Neste contexto, o presente estudo objetiva traçar o perfil epidemiológico de recém-nascidos atendidos em uma maternidade de referência para gestações em alto risco, podendo contribuir com o serviço em questão, identificando as demandas decorrentes do perfil dos atendimentos realizados.

\section{Materiais e métodos}

Foi realizado um estudo transversal, retrospectivo e descritivo, em uma maternidade referência para o SUS (Sistema Único de Saúde) no atendimento de alta complexidade da atenção à saúde da gestante e da criança, localizada na cidade de Blumenau-SC, inserida na Rede Cegonha (Portaria MS N 1.459/2011). A cidade de Blumenau possui aproximadamente 334.000 habitantes, nascendo em média 4.000 bebês a cada ano. A maternidade foco deste estudo não recebe apenas gestantes de alto risco, embora seja a referência regional do SUS para gestações de alto risco na macrorregião do Vale, atendendo majoritariamente a população de Blumenau e cidades próximas e recebendo gestantes transferidas via regulação estadual.

A população do estudo foi constituída por 3318 recém-nascidos atendidos na referência de alto risco no período de julho de 2017 a junho de 2018. As variáveis epidemiológicas do estudo: plano de saúde, idade materna, paridade, via de parto, idade gestacional, escore de APGAR, peso ao nascer, sexo e setor de encaminhamento pósnatal, foram coletadas do Livro de Registro de Nascimentos do Centro Obstétrico. Em algumas variáveis o número total foi reduzido devido à perda de dados durante a coleta 
de informações.

Os dados coletados foram organizados no software Excel e processados com a utilização do aplicativo Statistical Package for the Social Sciences (SPSS) para Windows, versão 17,0. A ausência de informações acerca de variáveis específicas (missing values) não acarretou a exclusão dos sujeitos da amostra.

Conforme a OMS, foram consideradas adolescentes as gestantes com idade até 19 anos, e prematuros os RN com idade gestacional inferior a 37 semanas (4). No grupo de prematuros, foram classificados como prematuridade extrema aqueles com idade gestacional inferior a 28 semanas, muito prematuros de 28 semanas até 32 semanas, prematuridade moderada de 32 a 34 semanas, e prematuros tardios de 34 semanas até 37 semanas de idade gestacional.

O Escore de APGAR no primeiro e quinto minuto de vida foi divido em: de 0-3 asfixia grave, 4-7 asfixia moderada e 8-10 boa vitalidade. Utilizou-se o Escore de APGAR $<4$ no quinto minuto como sendo um indicador de asfixia perinatal, já que este é considerado um dos critérios para o diagnóstico, segundo a Academia Americana de Pediatria (7).

Após o atendimento neonatal na sala de parto, os recém-nascidos foram encaminhados a três setores diferentes conforme suas condições clínicas: Alojamento Conjunto (AC), Unidade de Cuidado Intermediário Neonatal (UCIN) e Unidade de Terapia Intensiva Neonatal (UTI Neonatal).

Em atendimento à Resolução 196/96 do Conselho Nacional de Saúde/Ministério da Saúde, a utilização dos dados foi autorizada pelo Comitê de Ética da Instituição sob protocolo 099512/2018. Devido às características do estudo, foi dispensado a necessidade do termo de consentimento livre e esclarecido.

\section{Resultados}

Nesta análise, 3318 recém-nascidos foram atendidos na sala de parto do Hospital Santo Antônio entre o período de julho de 2017 e junho de 2018. Destes atendimentos, 20 foram natimortos, sendo 15 destes prematuros.

A maioria dos atendimentos foi realizada pelo SUS $(89,3 \%)$ e não tinha história de gestação prévia (41,2\%). O número máximo de gestações prévias em uma única gestante foi de 8 gestações, tendo este número se repetido em 4 oportunidades em outras gestantes. A via de parto mais prevalente foi a via vaginal, com 2042 casos $(61,5 \%)$. $61,6 \%$ das gestantes dos convênios de saúde realizaram parto cesárea, sendo esta mais prevalente ainda nas pacientes particulares, acontecendo em $72,5 \%$ dos partos. Já nas pacientes do SUS, o parto vaginal predomina em $64,5 \%$ das vezes.

A média encontrada para idade materna foi de 27,1 anos com Desvio Padrão (DP) de \pm 6,3 anos. A quantidade de mães adolescentes (até 19 anos) encontrada foi de 10,7\%. As gestantes com maior e menor idade respectivamente tiveram 56 e 12 anos.

A idade gestacional média em dias foi de aproximadamente 269 dias, com DP de \pm 17 dias. Os prematuros representaram $17 \%$ da amostra, somando um total de 544 pacientes, sendo que a maioria, 373 (68,6\%) destes são prematuros tardios (34-37 semanas).

O grupo de prematuros apresentou uma idade gestacional média de 239 dias, 171 $(31,4 \%)$ tinham idade gestacional menor de 34 semanas, 54\% nascidos de cesárea, $55,1 \%$ do sexo masculino, sendo a média de peso $1675 \mathrm{~g}$ com menor peso encontrado de $485 \mathrm{~g}$. A idade materna para estre grupo variou de 12 a 44 anos, com idade média de 28 anos. Quanto ao escore de APGAR mais prevalente visto nos prematuros, respectivamente no primeiro e quinto minuto foi 9 e 9 . Esses recém-nascidos foram em sua maioria (54\%) encaminhados para o Alojamento Conjunto, $17,7 \%$ foi para UCIN e 
$24 \%$ para a UTI Neonatal

Mais detalhes sobre os dados referentes ao perfil epidemiológico dos partos atendidos encontram-se na Tabela 1.

Tabela 1 - Perfil epidemiológico dos partos atendidos no Hospital Santo Antônio entre o período de julho de 2017 a junho de 2018.

\begin{tabular}{|c|c|c|c|}
\hline & $\mathbf{N}(\%)$ & Média $\pm D P$ & Mínimo/Máximo \\
\hline Planos de Saúde & $3318(100)$ & & \\
\hline SUS & $2964(89,3)$ & & \\
\hline Planos de Saúde - Convênios & $292(8,8)$ & & \\
\hline Particular & $62(1,9)$ & & \\
\hline Gestações Prévias & $3318(100)$ & & $0 / 8$ \\
\hline Sem gestação prévia & $1366(41,2 \%)$ & & \\
\hline 1 gestação prévia & $1014(30,4 \%)$ & & \\
\hline 2 gestações prévias & $518(15,6 \%)$ & & \\
\hline 3 gestações prévias & $235(7,1 \%)$ & & \\
\hline 4 gestações prévias & $109(3,3)$ & & \\
\hline 5 ou mais gestações prévias & $76(2,3 \%)$ & & \\
\hline Vias de Parto & $3318(100)$ & & \\
\hline Vaginal & $2042(61,5)$ & & \\
\hline Cesárea & $1276(38,5)$ & & \\
\hline Idade Materna Geral (anos) & $3318(100)$ & $27,1 \pm 6,3$ & $12 / 56$ \\
\hline Adolescentes (10-19 anos) & $355(10,7)$ & $17,7 \pm 1,4$ & $12 / 19$ \\
\hline Adultas (20-35 anos) & $2576(77,6)$ & $26,7 \pm 4,3$ & $20 / 35$ \\
\hline Maduras (> 35 anos) & $387(11,6)$ & $38,5 \pm 2,4$ & $36 / 56$ \\
\hline Idade Gestacional (dias) & $3196(100)$ & $\mathbf{2 7 0} \pm \mathbf{1 7 , 7}$ & $140 / 302$ \\
\hline Pré-termo* $(<37$ semanas/259 dias) & $544(17)$ & $239,7 \pm 19,8$ & $140 / 258$ \\
\hline Extremo $(<28$ semanas/196 dias $)$ & 26 & $180,1 \pm 10,8$ & $140 / 192$ \\
\hline $\begin{array}{l}\text { Muito extremo ( } 28-32 \text { semanas / } \\
196-224 \text { dias) }\end{array}$ & 59 & $210,5 \pm 7,9$ & $196 / 223$ \\
\hline Moderado (32-34 semanas/224-238 dias) & 86 & $231,6 \pm 4,3$ & $224 / 237$ \\
\hline Tardio (34-37 semanas/238-259 dias) & 373 & $250,4 \pm 6$ & $238 / 258$ \\
\hline A termo (37-42 semanas/259-294 dias) & $2643(82,7)$ & $276,1 \pm 8,4$ & $259 / 293$ \\
\hline Pós-termo (> 42 semanas/294 dias) & $9(0,3)$ & $296,2 \pm 2,8$ & $294 / 302$ \\
\hline
\end{tabular}

Abreviaturas: N, número de sujeitos amostrais; DP, desvio padrão.

* A idade gestacional pré-termo está subdividida em: extremo, muito extremo, moderada e tardio.

Conforme observado na Tabela 2, em relação ao perfil epidemiológico e o setor de encaminhamento após o nascimento dos recém-nascidos, observou-se leve predomínio do gênero masculino $(52,4 \%)$ em relação ao feminino $(47,6 \%)$. $\mathrm{O}$ grupo de extremo baixo peso $(<1000 \mathrm{~g})$ foi observado em 34 casos, com média de peso em $768 \pm 178 \mathrm{~g}$. Quarenta e dois foram os nascidos com muito baixo peso $(<1500 \mathrm{~g})$. Baixo peso $(<2500 \mathrm{~g})$ ocorreu em 363 pacientes $(11 \%)$. Nascidos com peso adequado ao nascimento representaram $86,8 \%$ dos casos.

Após atendidos pela equipe em sala de parto, 89,7\% dos recém-nascidos foram encaminhados para o Alojamento Conjunto, 4,8\% para a UCIN e 4,9\% para a UTI Neonatal.

Dos 163 recém-nascidos admitidos na UTI Neonatal, 134 (82,2\%) eram prematuros, os quais tinham idade gestacional variando entre 24 e 36 semanas, com uma média de 31 semanas (DP de \pm 3 semanas). A média de peso desses recém-nascidos que foram para UTI Neonatal foi de $1916 \mathrm{~g}$, variando entre $485 \mathrm{~g}$ a $4950 \mathrm{~g}$. Os outros 29 pacientes admitidos na UTI Neonatal eram a termo, com peso médio de $3073 \mathrm{~g}$, em sua maioria 
nascida de parto vaginal (62\%) e conforme o APGAR após o quinto minuto, $28 \%$ receberam APGAR $<7$.

De uma maneira geral, os escores de APGAR do primeiro e quinto minuto que com maior frequência apareceram foram respectivamente 9 e 10. Em 253 recém-nascidos o escore de APGAR foi menor que 7 no primeiro minuto de vida, e no quinto minuto de vida 40 recém-nascidos apresentaram esse mesmo escore. A asfixia perinatal, APGAR $<4$ no quinto minuto de vida, aconteceu em 18 recém-nascidos.

Tabela 2 - Perfil dos recém-nascidos no Hospital Santo Antônio entre o período de julho de 2017 a junho de 2018 e Setor de encaminhamento após Nascimento.

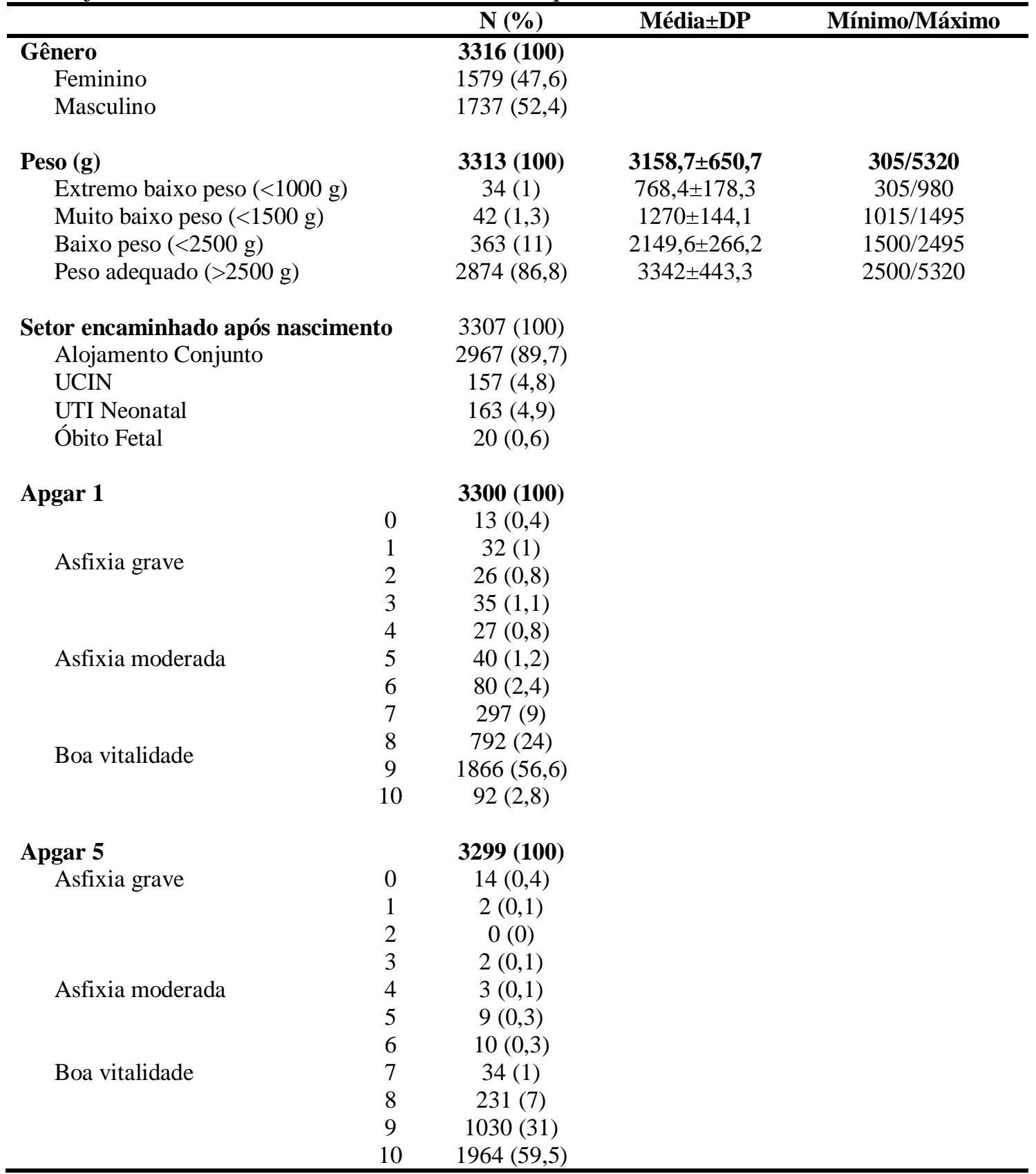

Abreviaturas: N, número de sujeitos amostrais; DP, desvio padrão; UCIN, Unidade de Cuidado Intermediário Neonatal; UTI Neonatal, Unidade de Terapia Intensiva.

\section{Discussão}


A Região de Saúde do Médio Vale do Itajaí, em Santa Catarina, é composta por 14 cidades. No ano de 2016 registrou 9.657 nascidos vivos, sendo a quarta maior região do Estado de Santa Catarina em número de nascidos vivos (8). Esta demanda evidencia a importância da maternidade estudada para o Estado de Santa Catarina, visto que registrou 3298 nascidos vivos no período de um ano entre 2017 e 2018 em que o estudo foi realizado, representando cerca de um terço (1/3) da população em questão.

A incidência de partos cesárea encontrada de 38,46\%. Segundo a OMS (9), taxas populacionais de cesárea de até $10-15 \%$ têm relação com diminuição da mortalidade materna e neonatal, e por outro lado, não existe associação entre taxas acima desses valores e redução da mortalidade. Sabe-se que a cesárea é uma intervenção efetiva para salvar a vida de mães e recém-nascidos, porém deve ter sua indicação precisa para esse benefício, pois pode causar complicações significativas e as vezes permanentes. $\mathrm{O}$ valor encontrado neste estudo pode estar relacionado ao fato de se tratar de uma unidade referência em gestação de alto risco.

Esta pesquisa demonstrou que $10,7 \%$ de gestantes eram adolescentes durante o período avaliado. Conforme o IBGE em 2006 no Brasil 20,6\% dos nascidos vivos eram filhos de mães com idade de 15 a 19 anos. Em 2012, dos 2.905 .789 milhões de nascidos vivos, 560.147 mil (19,3\%) foram de mães adolescentes (10). O dado encontrado mostra um menor índice de gravidez na adolescência na região estudada em comparação ao restante do país.

Condições como a prematuridade e o baixo peso ao nascer tendem a ser mais frequentes em adolescentes, levando consequentemente ao aumento dos índices de mortalidade infantil (11). No entanto, neste estudo não foi encontrada correlação significativa entre idade materna e as variáveis baixo peso e prematuridade.

A taxa de prematuridade encontrada neste serviço referência SUS de alta complexidade materno infantil é $17 \%$, é maior do que a apontada pela Pesquisa Nascer no Brasil: inquérito nacional sobre parto e nascimento, publicada pela Fiocruz (12) no ano de 2014 revelou que a taxa de prematuridade nacional é de $11,5 \%$.

A pesquisa aponta uma $55,1 \%$ dos prematuros eram do sexo masculino. Estudos realizados na Holanda demonstraram em seus resultados que o sexo masculino para o recém-nascido prematuro estava associado ao aumento do risco de sofrimento fetal, enquanto foi observado efeito protetor do sexo feminino (13), no entanto não foi o objetivo desse trabalho avaliar o risco de sofrimento fetal nos bebês.

O escore de APGAR é utilizado para identificar aqueles recém-nascidos que necessitam de cuidados adicionais. Um APGAR de 7-10 aponta para um recém-nascido sadio. No entanto um APGAR < 7 é um sinal de alerta de acordo com a alteração fisiopatológica e maturidade do concepto (13). O índice de APGAR <7 no primeiro minuto encontrado nessa pesquisa esteve presente em 253 (7,7\%) dos casos, já no quinto minuto, 98,8\% dos recém-nascidos apresentaram APGAR de 7 ou mais, indicando que mesmo que tenham apresentado dificuldades ao nascimento, na avaliação após 5 minutos houve boa recuperação, o que sugere um bom prognóstico e chance de sobrevida.

Do total de atendimentos, 163 pacientes necessitaram de encaminhamento para a UTI Neonatal provindo diretamente após o atendimento em sala de parto, sendo destes 134 prematuros, os quais possuem com frequência período prolongado de internação hospitalar. O serviço estudado possui 10 leitos disponíveis de UTI Neonatal habilitados pela Rede Cegonha. Além disso, este hospital recebe pacientes por outros mecanismos de admissão e transferências.

Esta pesquisa mostra uma alta incidência de prematuridade quando comparada à média brasileira e traça um perfil epidemiológico compatível com maternidade de referência em alto risco. Esses dados são expressivos já que refletem as condições de 
nascimento neonatais da Região do Médio Vale do Itajaí - SC, o que aponta para a necessidade de melhoria da assistência pré-natal, a fim de que possam ser identificados mais precocemente os fatores de risco que levam ao parto prematuro, e assim fornecida a atenção e seguimento especializado, sabida a íntima relação com a morbimortalidade infantil. Dentre as limitações da presente pesquisa, a não avaliação por completo dos dados referentes ao pré-natal realizado nesse grupo de gestantes dificulta um maior entendimento dos diversos fatores relacionados a prematuridade.

Embora a presente investigação não apresente elementos causais que possam predizer o desfecho de prematuridade, contribui significativamente na caracterização deste serviço de obstetrícia e no melhor entendimento do perfil dos sujeitos e atendimentos nos hospitais com abordagem similar. Por fim, torna-se evidente a necessidade de investimentos e planejamento estratégico adequado para o cuidado e atendimento ao recém-nascido de alto risco, incluindo a ampliação de leitos disponíveis em UTI Neonatal na região, conforme orientação do Departamento Científico de Neonatologia da Sociedade Brasileira de Pediatria, preconizando um mínimo de 4 leitos para cada 1000 nascidos vivos.

\section{Referências}

1. Brasil. Atenção à Saúde do Recém-Nascido: guia para os profissionais de saúde. In: Ministério da Saúde Secretaria de Atenção à Saúde [Internet]. Brasília; 2011 [citado 2019 Mar 17]. Disponível em: http://www.saude.gov.br/editora

2. Lansky S, Friche AA de L, da Silva AAM, Campos D, Bittencourt SD de A, de Carvalho ML, et al. Pesquisa Nascer no Brasil: perfil da mortalidade neonatal e avaliação da assistência à gestante e ao recém-nascido. Cad Saude Publica 2014; 30(1): 192-207.

3. Liu L, Johnson HL, Cousens S, Perin J, Scott S, Lawn JE, et al. Global, regional, and national causes of child mortality: an updated systematic analysis for 2010 with time trends since 2000. Lancet 2012; 379(9832): 2151-61.

4. Brasil. Pré-natal e Puerpério: atenção qualificada e humanizada. In: Ministério da Saúde Secretaria de Atenção à Saúde [Internet]. Brasília; 2005 [citado 2019 Mar 17]. Disponível em: http://bvsms.saude.gov.br/bvs/publicacoes/manual_pre_natal_puerperio_3ed.pdf

5. Blencowe H, Cousens S, Oestergaard MZ, Chou D, Moller A-B, Narwal R, et al. National, regional, and worldwide estimates of preterm birth rates in the year 2010 with time trends since 1990 for selected countries: a systematic analysis and implications. Lancet 2012; 379 (9832): 2162-72.

6. Matijasevich A, Silveira MF da, Matos ACG, Rabello Neto D, Fernandes RM, Maranhão AG, et al. Estimativas corrigidas da prevalência de nascimentos pré-termo no Brasil, 2000 a 2011. Epidemiol e Serviços Saúde 2013; 22(4): 557-64.

7. Procianoy RS, Silveira R de C. Síndrome hipóxico-isquêmica. J Pediatr (Rio J) 2001; 77 1): 63-70.

8. DATASUS M da S. Informações de Saúde. Nascidos vivos - Santa Catarina [Internet]. 2016 [citado 2019 Feb 20]. Disponível em: http://tabnet.datasus.gov.br/cgi/deftohtm.exe?sinasc/cnv/nvsc.def

9. OMS. Declaração da OMS sobre Taxas de Cesáreas [Internet]. Genebra; [citado 2019 Apr 10]. Disponível em: https://apps.who.int/iris/bitstream/handle/10665/161442/WHO_RHR_15.02_por.pdf; jsessionid=F7A8F72189349A94F4328A6AE9C28F1B?sequence $=3$

10. IBGE. Indicadores Sociodemográficos e de Saúde no Brasil [Internet]. 2009 [citado 2019 Mar 17]. Disponível em: https://www.ibge.gov.br/estatisticas-novoportal/sociais/saude/9336-indicadoressociodemograficos-e-de-saude-no-brasil.html?=\&t=o-que-e

11. Aquino-Cunha M, Queiroz-Andrade M, Tavares-Neto J, Andrade T. Gestação na Adolescência: Relação com o Baixo Peso ao Nascer. Rev Bras Ginecol e Obs 2002; 24 (8): 513-9.

12. ENSP. Nascer no Brasil: Inquérito nacional sobre parto e nascimento [Internet]. [citado 2019 Mar 17]. Disponível em: http://www6.ensp.fiocruz.br/nascerbrasil/

13. Cunha $\mathrm{A}$ de $\mathrm{A}$, Fernandes $\mathrm{D}$ de $\mathrm{S}$, Melo $\mathrm{PF}$ de, Guedes $\mathrm{MH}$. Fatores associados à asfixia perinatal. Rev Bras Ginecol e Obs 2004; 26 (10): 799-805. 